\title{
28 Resarch Soure Premorbid IL-6 levels may predict mortality from COVID-19
}

Morry Silberstein ( $\square$ m.silberstein@curtin.edu.au )

Curtin University https://orcid.org/0000-0003-4877-0672

\section{Short Report}

Keywords: IL-6, COVID-19, Vitamin D

Posted Date: May 29th, 2020

DOl: https://doi.org/10.21203/rs.3.rs-31814/v1

License: (9) This work is licensed under a Creative Commons Attribution 4.0 International License. Read Full License 


\section{Abstract}

Purpose: There is evidence that interleukin-6 (IL-6) levels are elevated in cases of complicated COVID-19, but it is also possible that this cytokine has a far more important role in the pathogenesis of viral infection. Methods: Data on COVID-19 mortality from Italy and the UK were compared with previously published results of mean IL- 6 levels from these countries as well as from the USA. Results: There was a highly significant correlation $(r=0.9883 ; p=0.00025)$ between age-stratified mortality rates and IL-6 levels from similar healthy individuals. Levels of IL-6 were proportionately higher in males, the elderly, individuals of black ethnicity and obese individuals, with similar findings in relation to COVID-19 mortality in these groups. Conclusions: IL-6 levels prior to infection may predict mortality, given that this cytokine directly facilitates viral cell entry and replication. This provides a rationale for prophylactic and therapeutic measures directed at lowering IL-6, including Vitamin D prescription.

\section{Introduction}

Previous studies have suggested that interleukin-6 (IL-6) levels are elevated in cases of complicated COVID-19 ${ }^{1}$, and that this cytokine may be a potential biomarker for disease progression ${ }^{2}$. While increased IL-6 production may well be a consequence of severe infection, could it also be a predisposing factor? COVID-19 requires cathepsin $L$ to infect pulmonary epithelial cells ${ }^{3}$, with cathepsin $L$ production upregulated by IL- $6^{4}$. This interleukin also likely upregulates expression of the ACE2 receptor which the virus utilizes for cellular entry ${ }^{5}$. If IL-6 levels prior to infection were found to correlate with disease severity, this might explain the documented poorer outcomes in males, with increasing age and in obese individuals ${ }^{6}$, given that levels of this cytokine are higher in males ${ }^{7}$, increase with advancing age ${ }^{8}$ and in obesity ${ }^{9}$. This might also explain why Vitamin $\mathrm{D}$ appears to be protective against severe infection ${ }^{10}$, given that IL-6 production is down regulated by this vitamin ${ }^{11}$ as well as the worse outcomes experienced by patients of African-American descent ${ }^{12}$, given that these individuals have higher IL- 6 levels than other population groups ${ }^{13}$.

I sought to determine whether IL-6 levels in healthy individuals from a similar demographic to patients infected with COVID-19 correlate with severity of clinical manifestations.

\section{Methods}

Given the range of severity of clinical manifestations of COVID-19, I limited my analysis to assessment of mortality. To limit any potential confounding factors, I searched the literature for results of IL-6 determinations in healthy individuals with included age and sex data from specific geographical regions for which published mortality rates from COVID-19 were available. The largest study, in terms of numbers of subjects, by Stowe and colleagues ${ }^{13}$ did not include detailed demographic information broken down into age groups, so I performed my analysis on data from an Italian study that examined IL-6 from the Tuscany region ${ }^{14}$. Data on COVID-19 mortality from Italy was sourced from statista.com ${ }^{15}$. I also 
examined published data on normal levels of IL- 6 broken down by ethnic minorities ${ }^{13}$ and Body Mass Index $(\mathrm{BMI})^{9}$ and compared these with a recently published study describing mortality by ethnic group and $\mathrm{BMI}^{6}$. Statistical analysis on age data was carried out using the Pearson Correlation Coefficient Calculator. Data on sex, ethnic minority status and BMI were insufficient for statistical analysis, and these were assessed qualitatively. Age data were stratified slightly differently in each study, with the majority quoting in increments of 10 years from 20 years of age upwards. Where included studies stratified age groups in different formats, ages were rounded to nearest multiple of 10 . For example, recently published UK data included a category of 18 to 40 years of age ${ }^{6}$; this category was rounded up to 20 to 40 in my analysis. Other studies, including that by Ferruci and colleagues ${ }^{14}$ included categories in the format " 20 to 39 " years of age, which was rounded up to 20 to 40 .

\section{Results}

The data on Italian mortality ${ }^{15}$ provided raw numerical deaths by population groups but did not provide information on stratified mortality rates by age group. To assess the validity of using this data, I compared these results with stratified mortality rate data in a large UK study ${ }^{6}$ (see Table 1). There was an extremely strong correlation between age stratified Italian raw numerical deaths and UK mortality rates $(r(6)=0.9837 ; 95 \% \mathrm{Cl}: 0.85-1.0 ; p=0.00396$, see Fig. 1$)$. Given the likely validity of using the Italian data, I compared this with IL-6 levels derived from a healthy Italian population ${ }^{14}$. There was an extremely strong correlation between age stratified Italian raw numerical deaths and IL- 6 levels $(r(6)=0.9883 ; 95 \%$ Cl: $0.89-1.0 ; p=0.00025$, see Table 2 and Fig. 2). As a broad test to confirm this, I also compared UK death rates with historic data published by Wei and colleagues ${ }^{16}$ on IL-6 levels in a similar population. Mean death rates in patients over 60 were more than 10 times higher than in patients under 50; mean levels of IL- 6 were higher in older patients, although only by $26 \%$ (Table 3). A similar discord was present in a comparison between UK death rates and a diagnosis of obesity, using IL-6 levels from a US study 9 . Mean death rates in non-obese patients were $48 \%$ higher than in non-obese patients; this compared with IL-6 levels more than 300 times greater in obese patients (Table 4). However, sex and ethnicity data demonstrated a much closer relationship between death rates and mean IL-6 levels. Male death rates were $72 \%$ higher than amongst females, compared to mean IL- 6 levels for males being $112 \%$ greater than mean female levels (Table 5). Similarly death rates amongst UK patients of black ethnicity were $40 \%$ higher than amongst white patients, compared with 59\% greater IL-6 levels amongst black patients derived from a US study ${ }^{13}$ (Table 6).

\section{Discussion}

This is the first study to have demonstrated a significant correlation between IL-6 levels in matched healthy individuals and age-stratified mortality from COVID-19. Despite the format of data available for other variables deemed to be risk factors for increased mortality including sex, obesity and ethnicity ${ }^{6}$, not lending itself to statistical analysis, premorbid IL- 6 in matched populations had similar higher levels when 
compared with mortality rates based on these risk factors. This suggests that IL- 6 may have a far more important role in the pathogenesis of viral infection than previously thought. IL- 6 upregulates cathepsin L production ${ }^{4}$, which then cleaves the virus, enabling it to infect pulmonary epithelial cells ${ }^{3}$. IL- 6 also upregulates the Angiotensin II AT1 receptor ${ }^{17}$, and likely upregulates pulmonary epithelial cell ACE2 receptors, with a positive correlation between IL- 6 and ACE2 levels in bronchioalveolar lavage (BAL) in patients with severe acute pulmonary inflammation ${ }^{5}$. Viral reproduction within pulmonary epithelial cells then recruits inflammatory cells which, in turn produce cytokines (as part of the "cytokine storm" phenomenon ${ }^{18}$ ) including IL-6. This could be expected to set up a noxious cycle between immune cells and pulmonary epithelium which the virus utilizes to enter cells and replicate. The principal predisposing factor could well be IL-6, with premorbid levels correlating with stratified mortality rates. There are other manifestations of this infection that could readily be attributed to IL-6. Levels of this interleukin are generally low in children, but there is a short spurt in early childhood ${ }^{19}$, correlating with a recently described severe COVID-19 manifestation in young children, a Kawasaki-like condition ${ }^{20}$. IL-6 is also thrombogenic, which may well account for this significant cause of death from the virus ${ }^{21}$. Intense exercise also produces large quantities of IL-622, which may account for the more severe clinical manifestations of infection amongst athletes ${ }^{23}$.

How can this be utilized to reduce mortality? The role of Vitamin D deficiency as a predictor for worse clinical outcomes remains the focus of investigation, although early results appear to support this contention ${ }^{10}$. What is unclear is whether Vitamin $D$ exerts a general immune-enhancing effect, or whether it may have a specific role in prevention and treatment of COVID-19. The results herein reported point strongly to the latter, given that Vitamin D lowers immune cell production of IL- $6^{11}$. In addition to Vitamin D prescription, both prophylactic, and, potentially as a specific therapy ${ }^{18}$, there may well be other therapeutic strategies which could be deployed, including nutritional supplementation, such as ingesting Brazil nuts ${ }^{24}$, and administration of drugs which lower IL-6 production such as doxycycline ${ }^{25}$ and budesonide/formoterol ${ }^{26}$.

While the results of this work offer a rationale for the diverse clinical manifestations of COVID-19 and a range of therapeutic options for trial, this must be tempered by the limitations of my analysis. The data analysed were not strictly comparable, with premorbid data on IL-6 levels derived from different populations to those with mortality data. Further, only the age-stratified results are supported by statistical analysis as the other data on other factors including sex, ethnicity and obesity could not be assessed directly for statistical significance. While substantial premorbid data on IL-6 levels may never be obtainable in patients who die from COVID-19, prospective trials of therapeutic measures can certainly be undertaken, but, given that Vitamin D causes little harm if administered under clinical supervision, this study certainly supports its widespread prescription.

In conclusion, this study has demonstrated a highly significant correlation between premorbid IL-6 levels and mortality rates from COVID-19, accounting for a range of previously described clinical predictors and, potentially directing future therapeutic strategies. 


\section{Declarations}

Competing Interests: None

Funding Source: None

\section{References}

1. Coomes EA, Haghbayan H. Interleukin-6 in COVID-19: A systematic review and meta-analysis. medRxiv. 2020. doi:https://doi.org/10.1101/2020.03.30.20048058

2. Ulhaq ZS, Soraya GV. Interleukin-6 as a potential biomarker of COVID-19 progression. Med Mal Infect. 2020; 50:382-383. doi:10.1016/j.medmal.2020.04.002

3. Huang IC, Bosch BJ, Li F, et al. SARS coronavirus, but not human coronavirus NL63, utilizes cathepsin L to infect ACE2-expressing cells. J Biol Chem. 2006; 281:3198-203.

4. Gerber A, Welte T, Ansorge S, Bühling F. Expression of cathepsins $B$ and $L$ in human lung epithelial cells is regulated by cytokines. Adv Exp Med Biol. 2000; 477:287-92.

5. Schouten LR, van Kaam AH, Kohse F, et al. Age-dependent differences in pulmonary host responses in ARDS: a prospective observational cohort study. Ann Intensive Care. 2019; 9:55.

6. Williamson E, Walker AJ, Bhaskaran K, et al. OpenSAFELY: factors associated with COVID-19-related hospital death in the linked electronic health records of 17 million adult NHS patients. medRxiv. 2020. doi: https://doi.org/10.1101/2020.05.06.20092999

7. Liu X, Xiao Y, Zhu Q, et al. Abstract 595: Gender difference was present in the production of cytokines in mice with PM Exposure. Art Thromb Vasc Biol. 2017; 37:A595.

8. Wei J, Xu H, Davies JL, Hemmings GP. Increase of plasma IL-6 concentration with age in healthy subjects. Life Sci. 1992; 51:1953-6.

9. Khaodhiar L, Ling PR, Blackburn GL, Bistrian BR. Serum levels of interleukin-6 and C-reactive protein correlate with body mass index across the broad range of obesity. JPEN J Parenter Enteral Nutr. 2004; 28:410-5.

10. Ilie PC, Stefanescu S, Smith L. The role of vitamin D in the prevention of coronavirus disease 2019 infection and mortality. Aging Clin Exp Res. 2020; 1-4. doi:10.1007/s40520-020-01570-8

11. Sadeghi K1, Wessner B, Laggner $U$, et al. Vitamin D3 down-regulates monocyte TLR expression and triggers hyporesponsiveness to pathogen-associated molecular patterns. Eur J Immunol. 2006; 36:361-70.

12. Yancy CW. COVID-19 and African Americans. JAMA. 2020; 323:1891-1892. doi:10.1001/jama.2020.6548

13. Stowe RP, Peek MK, Cutchin MP, Goodwin JS. Plasma cytokine levels in a population-based study: relation to age and ethnicity. J Gerontol A Biol Sci Med Sci. 2010; 65:429-33. doi: 10.1093/gerona/glp198. 
14. Ferrucci L, Corsi A, Lauretani Fet al. The origins of age-related proinflammatory state. Blood. 2005; 105:2294-9.

15. https://www.statista.com/statistics/1105061/coronavirus-deaths-by-region-in-italy/ (to 13/05/2020, accessed 19/05/2020)

16. Wei J, Xu H, Davies JL, Hemmings GP. Increase of plasma IL-6 concentration with age in healthy subjects. Life Sci. 1992; 51:1953-6.

17. Senchenkova EY, Russell J, Yildirim A, Granger DN, Gavins FNE. Novel role of T Cells and IL-6 (Interleukin-6) in angiotensin II-induced microvascular dysfunction. Hypertension. 2019; 73:829-838.

18. Silberstein M. Vitamin D: A simpler alternative to tocilizumab for trial in COVID-19? Med Hypotheses 2020; 109767.

19. Sack U, Burkhardt U, Borte M, Schädlich H, Berg K, Emmrich F. Age-dependent levels of select immunological mediators in sera of healthy children. Clin Diagn Lab Immunol. 1998; 5:28-32.

20. Viner RM, Whittaker E. Kawasaki-like disease: emerging complication during the COVID-19 pandemic. Lancet. 2020 May 13. doi: 10.1016/S0140-6736(20)31129-6

21. Divani AA, Andalib S, Di Napoli M, et al. Coronavirus disease 2019 and stroke: clinical manifestations and pathophysiological insights [published online ahead of print, 2020 May 12. J Stroke Cerebrovasc Dis. 2020; 104941. doi:10.1016/j.jstrokecerebrovasdis.2020.104941

22. Pedersen BK, Steensberg A, Schjerling P. Exercise and interleukin-6. Curr Opin Hematol. 2001; 8:13741. doi: $10.1097 / 00062752-200105000-00002$.

23. Matricardi P, Dal Negro R, Nisini R. The first, comprehensive immunological model of COVID-19: implications for prevention, diagnosis, and public health measures. Preprints. 2020. 2020040436.

24. Thomson CD, Chisholm A, McLachlan SK, Campbell JM. Brazil nuts: an effective way to improve selenium status. Am J Clin Nutr. 2008; 87:379-84.

25. Conforti C, Giuffrida R, Zalaudek I, Di Meo N. Doxycycline, a widely used antibiotic in dermatology with a possible anti-inflammatory action against IL-6 in COVID-19 outbreak. Dermatol Ther. 2020: e13437.

26. Suda K, Tsuruta M, Eom J, et al. Acute lung injury induces cardiovascular dysfunction: effects of IL-6 and budesonide/formoterol. Am J Respir Cell Mol Biol. 2011; 45:510-6.

\section{Tables}

Table 1. Age and death data from Italy and UK populations 


\begin{tabular}{|l|l|l|}
\hline Age & Italy Deaths & UK Deaths/mill \\
\hline $20-40$ & 67 & 6.7 \\
\hline $40-50$ & 257 & 32.7 \\
\hline $50-60$ & 1051 & 115.7 \\
\hline $60-70$ & 3107 & 288.1 \\
\hline $70-80$ & 8196 & 800.8 \\
\hline $80+$ & 16842 & 2587.2 \\
\hline
\end{tabular}

Legend: Age-stratified deaths in Italy correlate strongly with UK death rates

Table 2. Mean IL-6 levels by agge and death data from Italy

\begin{tabular}{|l|l|l|}
\hline Age & IL-6 (pg/ml) & Italy Deaths \\
\hline $20-40$ & 0.6 & 67 \\
\hline $40-50$ & 0.9 & 257 \\
\hline $50-60$ & 1.0 & 1051 \\
\hline $60-70$ & 1.2 & 3107 \\
\hline $70-80$ & 1.7 & 8196 \\
\hline $80+$ & 2.6 & 16842 \\
\hline
\end{tabular}

Legend: Mean IL-6 levels correlate strongly with deaths in Italy

Table 3. Mean IL-6 levels by age and death rates from UK

\begin{tabular}{|l|l|l|}
\hline Age & IL-6 $(\mathrm{pg} / \mathrm{ml})$ & UK Death Rate/million \\
\hline$<50$ & 1.9 & 15.1 \\
\hline$>60$ & 2.4 & 351.4 \\
\hline
\end{tabular}

Legend: Mean IL-6 levels and death rate both higher in older individuals

Table 4. Mean IL-6 levels by Body_Mass Index and death rates from UK

\begin{tabular}{|l|l|l|}
\hline BMI & IL-6 & UK Death Rate/million \\
\hline$<30$ & 0.1 & 334.4 \\
\hline$>30$ & 3.2 & 496.8 \\
\hline
\end{tabular}


Legend: Mean IL-6 levels and death rate both higher in obese populations

Table 5. Mean IL-6 levels by sex and death rates from UK

\begin{tabular}{|l|l|l|}
\hline Sex & IL-6 $(\mathrm{pg} / \mathrm{ml})$ & UK Death Rate/million \\
\hline Male & 2.1 & 412.3 \\
\hline Female & 1.0 & 240.3 \\
\hline
\end{tabular}

Legend: Mean IL-6 levels and death rate both higher in males

Figures

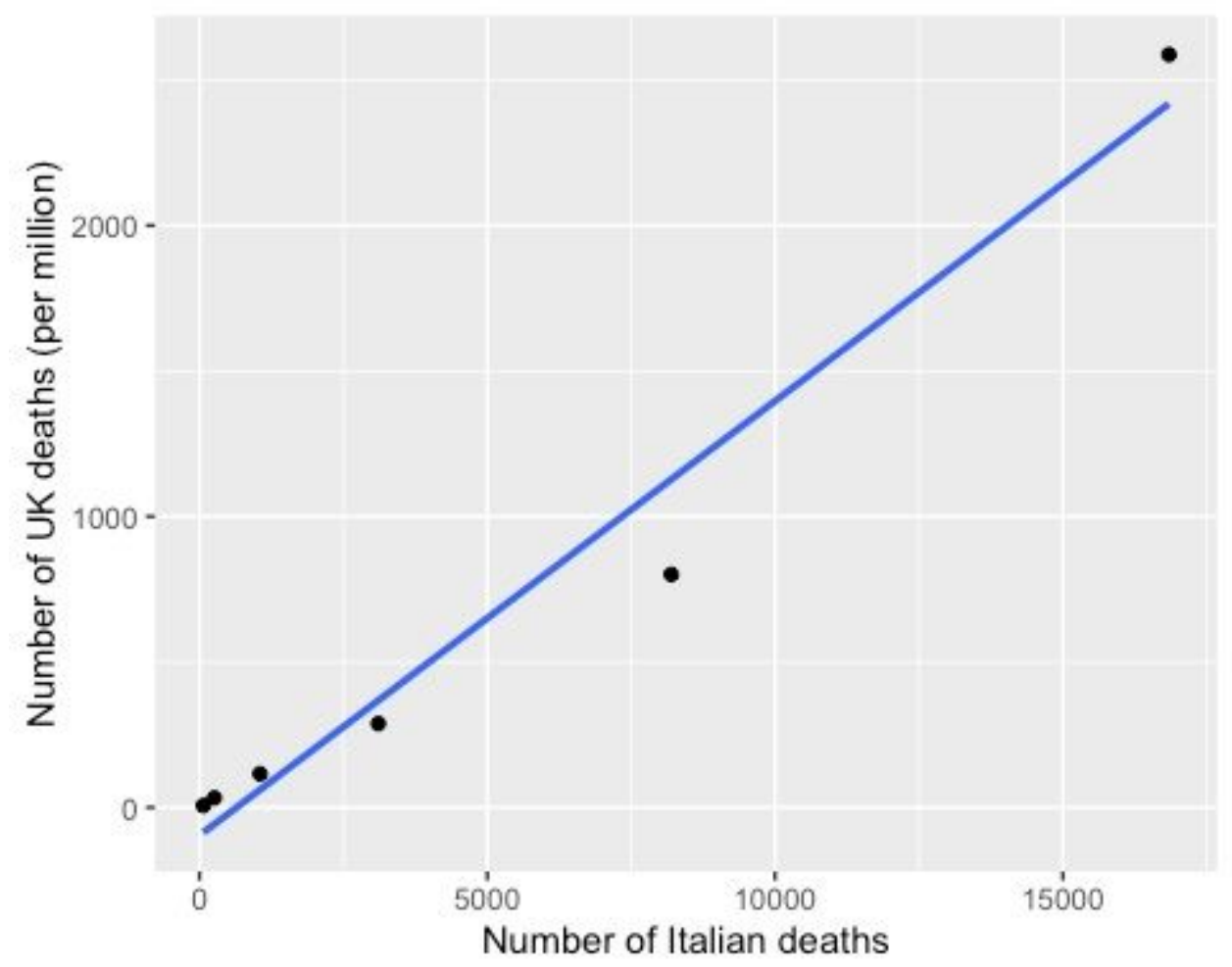

\section{Figure 1}

Age and death data from Italy and UK populations.Legend: Age-stratified deaths in Italy correlate strongly with UK death rates 


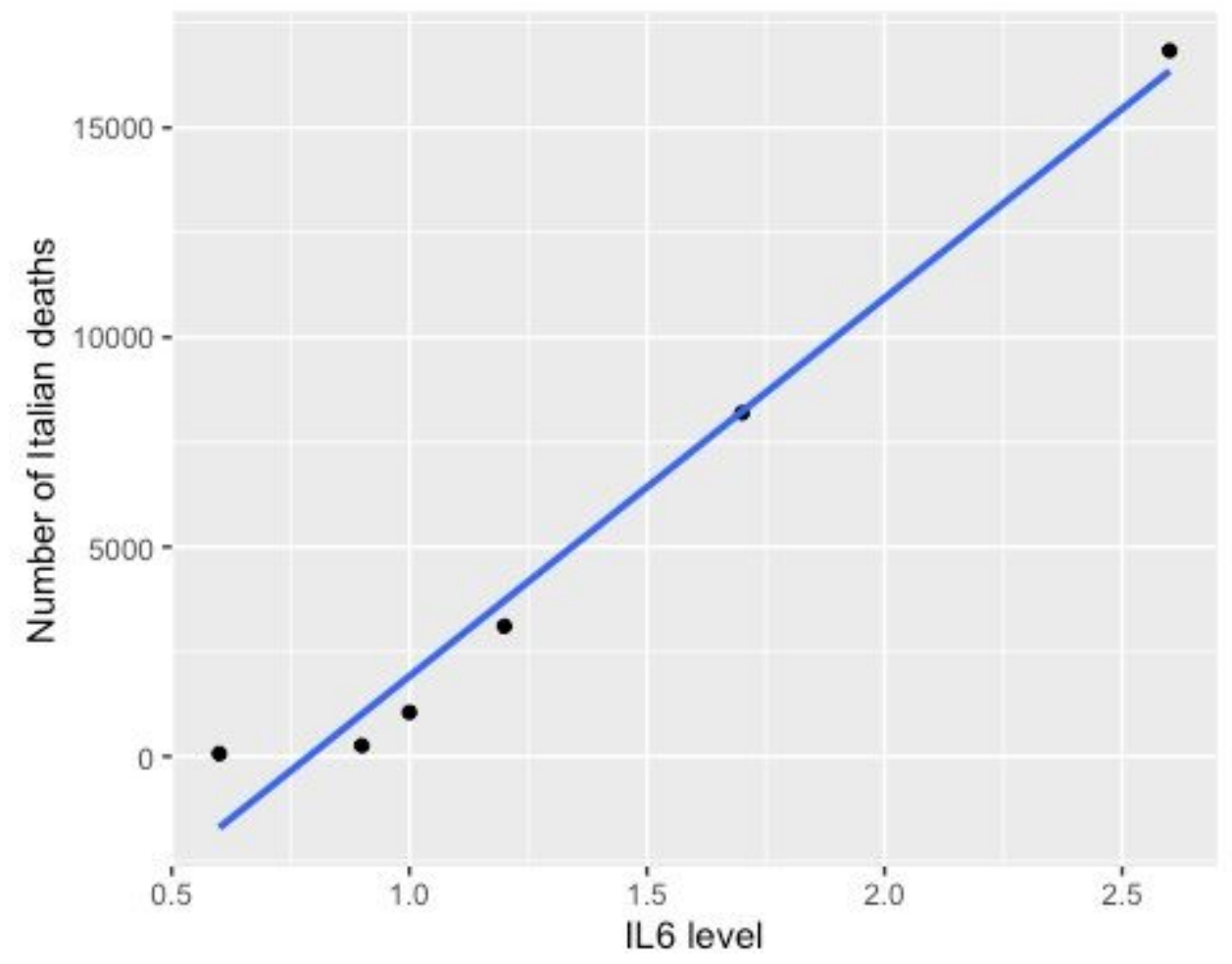

Figure 2

Mean IL-6 levels by age and death data from Italy. Mean IL-6 levels correlate strongly with deaths in Italy 